\title{
A Branching Streamer Propagation Argon Plasma Plume
}

\author{
Qing Xiong, Anton Y. Nikiforov, Xin Pei Lu, Senior Member, IEEE, and Christophe Leys
}

\begin{abstract}
Cold atmospheric-pressure plasma plumes have obtained great interests for their attractive features and application potentials. In this paper, a pulsed argon plasma plume was generated in the open air. Characteristic propagation of the argon plasma plume was carried out through high-speed imaging by an ICCD camera. An interesting propagation process was observed for the plasma plume, during which the plasma first propagated in a single streamer channel, and then with side branching as the applied voltage increased. The side branches are generated surrounding the main plasma channel but not split from the anode tip. This branching streamer propagation behavior was much different from the bulletlike development of typical plasma jets or streamers in point-wire or point-plane gaps.
\end{abstract}

Index Terms-Atmospheric-pressure plasma plumes, highspeed imaging, side branches, streamer propagation.

A S NOVEL plasma sources, cold atmospheric-pressure plasma plumes (C-APPPs) have recently obtained increasing attention for their unique features, such as chamberless operation and stable remote plasma generation. These attractive features make them prospective in several emerging novel applications, such as plasma biomedicine. Over the past years, many types of C-APPPs have been developed and investigated. Temporal and spatial characterization showed that the plasma plumes are propagating in the form of fast-moving bulletlike plasma volumes with velocities in the range of $10^{4}-10^{5} \mathrm{~ms}^{-1}$, which are typically more than three orders of magnitude in comparison to the working gas speed. However, mechanisms responsible for the production of C-APPPs remain unknown. Presently, there are two possible explanations, i.e. photoionization effects and ionization wave, contributing for the high-speed moving bulletlike plasma volumes. In this paper, a different

Manuscript received November 25, 2010; revised May 6, 2011 and May 8, 2011; accepted May 16, 2011. Date of publication July 12, 2011; date of current version November 9, 2011. This work was supported in part by the China Scholarship Council (CSC) and Interuniversity Attraction Poles Program of the Belgian Science Policy (project P6/08-PSI)

Q. Xiong is with the College of Electrical and Electronic Engineering, Huazhong University of Science and Technology, Wuhan 430074, China, and also with the Research Unit Plasma Technology, Department of Applied Physics, Ghent University, 9000 Ghent, Belgium (e-mail: xiongplasma@ hotmail.com).

A. Y. Nikiforov and C. Leys are with the Research Unit Plasma Technology, Department of Applied Physics, Ghent University, 9000 Ghent, Belgium (e-mail: anton-nik@yandex.ru; christophe.leys@ugent.be).

X. P. Lu is with the College of Electrical and Electronic Engineering, Huazhong University of Science and Technology, Wuhan 430074, China (e-mail: luxinpei@hotmail.com).

Color versions of one or more of the figures in this paper are available online at http://ieeexplore.iee.org.

Digital Object Identifier 10.1109/TPS.2011.2157922
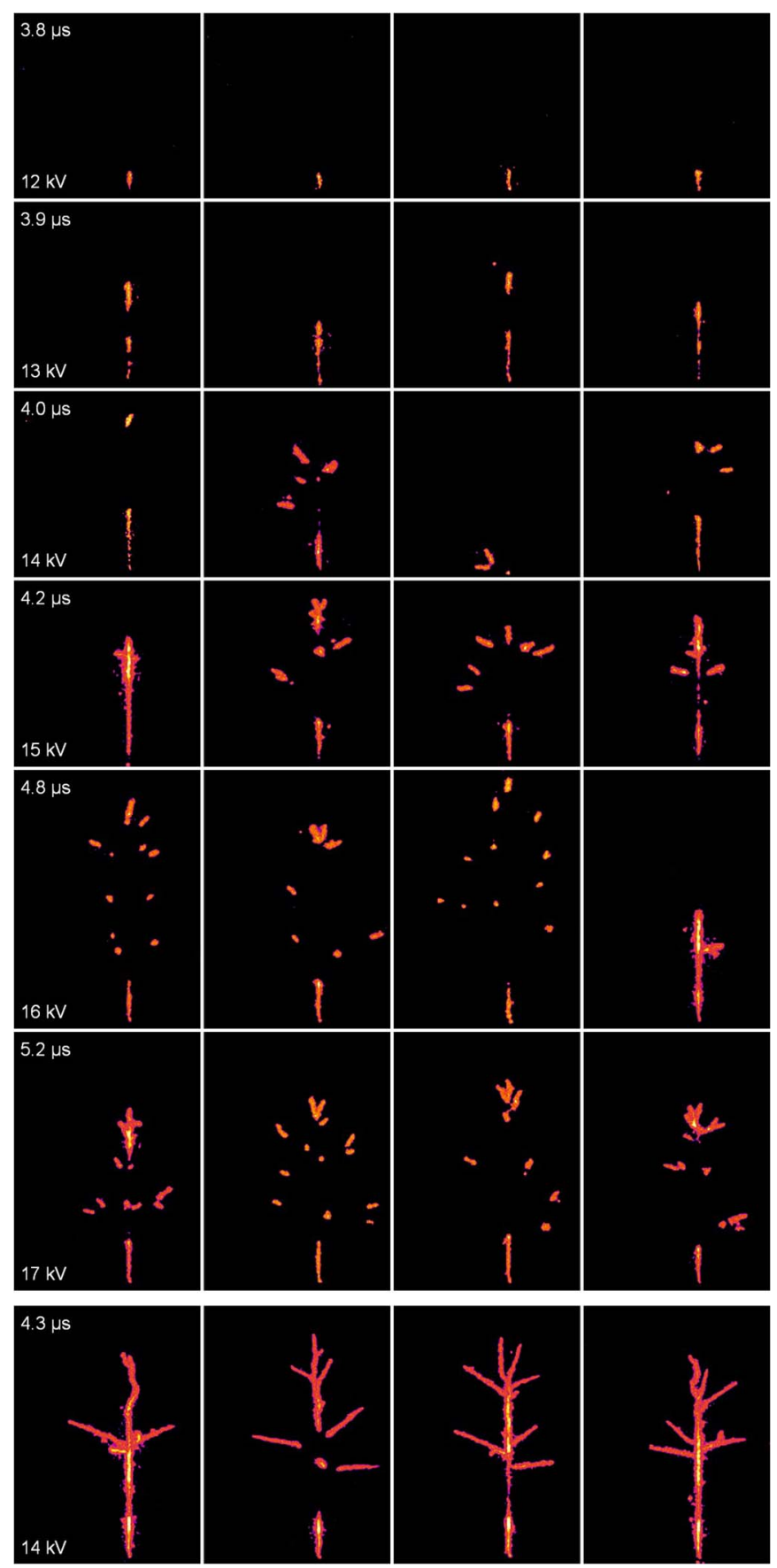

Fig. 1. High-speed image sequences of the argon plasma plume in the open air. The time delay and voltage values were marked in each first subimage of every image row, but the values apply to the entire row. For all images, except for the last image row (100-ns exposure time), the exposure time was set at $20 \mathrm{~ns}$. The nozzle exit (not marked) is located at the bottom of each image. 
propagation behavior of an atmospheric-pressure argon plasma plume was reported based on high-speed ICCD imaging.

The plasma plume setup is composed of a medical needle and powered by a high-voltage pulse generator. The applied pulsed voltage was set at $20 \mathrm{kV}$ at a repetition frequency of $2 \mathrm{kHz}$. Argon gas with $100 \mathrm{sccm}$ was used as the working gas. The temporal and spatial resolved emission imaging of the plasma plume was captured by a Hamamatsu ICCD camera (C8484). The ICCD camera gating time and the onset of applied voltage pulses were synchronized by a delay generator. Note that, due to the nonreproducibility of discharge structures, all the timeintegrated images presented here were captured in only one single shot. In addition, for each time delay, four typical images were presented in a row to show different structures of the discharges, as shown in Fig. 1.

The time delays marked in the top left of every image row were the corresponding imaging moments relative to the onset of voltage pulses. The corresponding applied voltage for each time delay was also shown therein. As can been seen, the plasma first propagated in a single primary streamer channel. As the applied voltage increased, small side streamer branches started to appear. The number of side branches was increased with the applied voltage. It is shown that there was always a primary streamer channel that originated from the needle tip and side branches were surrounding this primary channel. The side streamer branches were not extending from the primary streamer but generated and propagating surrounding it. This is much clear when the imaging exposure time increased, as shown in the last image-row (exposure time $100 \mathrm{~ns}$ ). These propagation processes are much different to that of typical plasma jets; in the latter case, the plasma propagated in the form of bulletlike plasma volumes without any side branching [1].
It is also different in comparison with typical positive streamers generated in point-wire or point-plane gaps. For typical positive streamers, streamer branching is generated around the anode tip [2]. In our case, it was mostly observed surrounding the primary streamer channel, precisely speaking, the argon gas stream.

It can be shown that the high-speed images represent a mapping of the spatial distribution of the relevant excited species in the plasma. From optical emission spectroscopy, it was shown that emissions were mainly from excited $\mathrm{Ar}^{*}$ and $\mathrm{N}_{2}^{*}$. The primary streamer channel was supposed to be formed by the excited argon species, which can be generated under the high external electric field. This is also why the primary streamer channel always propagated from the needle exit and followed the gas stream [3]. The generated side branches are probably related to the diffused air surrounding the argon gas stream. It is possible because the air concentration increased for the space away from the gas stream axis and nitrogen molecules can be easily excited by electrons which can be generated due to several effects, such as high external electric field and photoionization processes.

\section{REFERENCES}

[1] M. Teschke, J. Kedzierski, E. G. Finantu-Dinu, D. Korzec, and J. Engemann, "High-speed photographs of a dielectric barrier atmospheric pressure plasma jet," IEEE Trans. Plasma Sci., vol. 33, no. 2, pp. 310-311, Apr. 2005.

[2] S. Nijdam, F. Wetering, R. Blanc, E. Veldhuizen, and U. Ebert, "Probing photo-ionization: Experiments on positive streamers in pure gases and mixtures," J. Phys. D, Appl. Phys., vol. 43, no. 14, p. 145 204, Apr. 2010.

[3] Q. Xiong, A. Y. Nikiforov, X. P. Lu, and C. Leys, "High-speed dispersed photographing of an open-air argon plasma plume by a grating-ICCD camera system," J. Phys. D, Appl. Phys., vol. 43, no. 41, p. 415201 , Oct. 2010 . 